\title{
Clinical Presentation and Frequency of Metabolic Syndrome in Women With Polycystic Ovary Syndrome: An Experience From a Tertiary Care Hospital in Pakistan
}

\author{
Sarwat Anjum ${ }^{1}$, Saima Askari ${ }^{2}$, Musarrat Riaz ${ }^{1}$, Abdul Basit ${ }^{2}$ \\ 1. Endocrinology, Baqai Institute of Diabetology and Endocrinology, Karachi, PAK 2. Medicine, Baqai Institute of \\ Diabetology and Endocrinology, Karachi, PAK
}

Corresponding author: Sarwat Anjum, drriakhan94@gmail.com

Review began 10/14/2020 Review ended 11/15/2020 Published 12/02/2020

\section{() Copyright 2020}

Anjum et al. This is an open access article distributed under the terms of the Creative Commons Attribution License CC-BY 4.0., which permits unrestricted use, distribution, and reproduction in any medium, provided the original author and source are credited.

\begin{abstract}
Introduction: Polycystic ovary syndrome (PCOS) is a common endocrine disorder affecting women of reproductive age. The aim of this study was to determine the variations in the clinical presentation and frequency of metabolic syndrome (MetS) in women with PCOS.
\end{abstract}

Methods: This cross-sectional study was conducted at the Baqai Institute of Diabetology and Endocrinology, Baqai Medical University, Karachi, Pakistan, from April 2019 to March 2020. Women attending the endocrine clinic who satisfied the Rotterdam criteria of PCOS and agreed to participate in the study were included. Detailed personal and family history of menstrual cycle, hirsutism, diabetes, hypertension, dyslipidemia and obesity was noted along with measurement of vitals, anthropometric measures and calculation of the body mass index. Physical examination performed for signs of hyperandrogenism, insulin resistance and biochemical and hormonal evaluation was also carried out in recruited participants. Statistical analysis was done using the Statistical Package for the Social Sciences (SPSS) version 20 (IBM Corp., Armonk, NY).

Results: A total of 153 participants with mean age of $27.2 \pm 8.13$ years were included in this study. Regarding clinical presentation, menstrual irregularity (oligomenorrhea 39.85\%, amenorrhea 38.9\%), followed by hirsutism 52.3\%, was the most common presentation. Polycystic appearance of ovaries was noted in 33.3\% of our study participants. MetS was identified in $46.4 \%$ participants (obesity was noted at the highest frequency at $82.4 \%$ followed by dyslipidemia at $56.2 \%)$.

Conclusion: We observed a high frequency of MetS in females presenting with PCOS. There is a need to evaluate women with PCOS for various components of MetS to prevent potential complications.

Categories: Endocrinology/Diabetes/Metabolism, Internal Medicine, Public Health

Keywords: pcos, metabolic syndrome, clinical presentation

\section{Introduction}

Polycystic ovary syndrome (PCOS) is one of the most common endocrine disorders that affects $5 \%-10 \%$ women of reproductive age [1,2]. It is clearly heterogeneous with speculative etiology, causing a wide range of reproductive, metabolic, endocrine and psychological effects. Among them, ovulatory dysfunction, menstrual irregularities, infertility, hyperandrogenism, increased insulin level, obesity, obstructive sleep apnea, nonalcoholic fatty liver disease, eating and mood disorders, cardiovascular disease (CVD) and an increased risk of type 2 diabetes mellitus (T2DM) are significant factors [3,4]. However, the most common presenting problem observed in PCOS is menstrual cycle disturbances (oligo/amenorrhea), hirsutism, infertility, dyslipidemia and metabolic disturbances due to insulin resistance (IR) [5]. Hyperinsulinemia, with consequent IR, has a significant role to play in its pathogenesis [6]. The diagnosis of PCOS is based on the presence of at least two of the three criteria, namely, chronic anovulation, hyperandrogenism (clinical or biological) and polycystic ovaries on ultrasound, according to Rotterdam criteria [2], which are widely used for the diagnosis of PCOS [6], in addition to excluding all other potential causes.

The prevalence of metabolic syndrome (MetS) is increasing throughout the world [7]. MetS and PCOS share a bidirectional relationship and common pathogenic factors that predispose women with PCOS to an increased risk of developing MetS [8]. Women with PCOS have a fivefold increase risk of developing MetS compared with women without PCOS, suggesting PCOS alone is an independent risk factor for MetS [9]. In one study, the reported prevalence of MetS was $44.6 \%$ in PCOS women [10]. MetS is a constellation of disorders that include abdominal obesity, impaired glucose tolerance/DM, hypertension and/or dyslipidemia [11]. MetS predispose patients with PCOS to high risk of CVD, T2DM and gynecological cancer [11]. PCOS and MetS share a bidirectional relationship and both conditions produce profound effect on fertility, reproductive biology and oxidative stress-induced vascular complications [8]. 
In a study conducted in Karachi, Pakistan, the reported prevalence of MetS in women with PCOS was $35.6 \%$, which was significantly greater than the control group [12]. This substantial risk of increased morbidity and mortality has long-term implications for health of women with PCOS [13]. Data regarding the association of MetS and PCOS is scarce in our part of the world; therefore, our objective was to determine the clinical presentation and frequency of MetS in women presenting with PCOS at a tertiary care hospital [4].

\section{Materials And Methods}

This cross-sectional study was conducted at the Baqai Institute of Diabetology and Endocrinology (BIDE), Baqai Medical University, Karachi, Pakistan, over a period of one year from April 1, 2019, to March 31, 2020. All 13- to 45 -year-old females attending the outpatient department satisfying the Rotterdam criteria of PCOS (defined as the presence of two of the following: oligo/anovulation, hyperandrogenism and/or polycystic ovaries on ultrasound) were recruited through non-probability consecutive sampling after taking informed verbal consent. Other causes of hyperandrogenism and menstrual irregularity were excluded by doing relevant lab investigations. Ethical approval was taken from the Institutional Review Board of BIDE.

Detailed personal, menstrual and family history of hypertension, diabetes, dyslipidemia and obesity (MetS) was taken on a predesigned proforma. MetS by definition comprises three or more of the following: (1) central obesity with waist circumference $\geqslant 80 \mathrm{~cm}$ (WHO cutoff for Asian women), (2) systolic blood pressure $\geqslant 130 \mathrm{mmHg}$ or diastolic blood pressure $\geqslant 85 \mathrm{mmHg}$ or taking antihypertensive medication, (3) fasting plasma glucose $\geqslant 100 \mathrm{mg} / \mathrm{dl}$ or previously diagnosed type 2 diabetes,

(4) plasma high-density lipoprotein (HDL) cholesterol $<50 \mathrm{mg} / \mathrm{dl}$, (5) plasma triglycerides $>150 \mathrm{mg} / \mathrm{dl}$ (as per American Heart Association/National Heart, Lung, and Blood Institute [AHA/NHLBI] criteria) [14]. Detailed physical examination was done including the measurement of blood pressure, pulse, waist circumference, and BMI. Signs of insulin resistance (acanthosis nigricans, skin tags) and signs of hyperandrogenism (hirsutism, acne, alopecia, clitoromegaly) were noted. The Ferriman-Gallwey score was used to assess the hirsutism (score $>8$ was considered positive). The biochemical and hormonal evaluation, including fasting plasma glucose, insulin, lipid profile, thyroid stimulating hormone, follicle stimulating hormone, luteinizing hormone, prolactin, estradiol, testosterone, 17-hydroxyprogesterone, dehydroepiandrosterone level, was carried out. The homeostatic model assessment of insulin resistance (HOMA-IR) was used to calculate insulin resistance: $\mathrm{HOMA}-\mathrm{IR}=$ glucose fasting $(\mathrm{mg} / \mathrm{dl})$ x fasting insulin $(\mu \mathrm{UI} / \mathrm{ml}) \div 405$; values above 1.5 are indicative of insulin resistance. Pelvic ultrasound was performed for the evaluation of ovarian morphology.

Data analysis was done using the Statistical Package for the Social Sciences (SPSS) version 20 (IBM Corp., Armonk, NY) to compute mean, standard deviation, and percentages.

\section{Results}

A total of 153 participants with a mean age of $27.2 \pm 8.13$ years were included in this study. The mean age of menarche was $12.68 \pm 1.2$ years while the mean BMI was found to be $31.68 \pm 7.37\left(\mathrm{~kg} / \mathrm{m}^{2}\right)$. The baseline characteristics and biochemical parameters are mentioned in Table 1 . 


\section{Cureus}

\section{Parameters}

$\mathrm{N}$

Age (years)

Age at menarche (years)

Marital status

Single

Married

BMI $\left(\mathrm{kg} / \mathrm{m}^{2}\right)$

Waist circumference (cm)

Systolic blood pressure $(\mathrm{mmHg})$

Diastolic blood pressure $(\mathrm{mmHg})$

Fasting insulin

Fasting blood sugar (mg/dl)

HOMA-IR

FSH

LH

\section{Estradiol}

Testosterone

TSH

Prolactin
Mean \pm SD or N (\%) or median (IQR)

153

$27.2 \pm 8.13$

$12.68 \pm 1.2$

$65(43.9 \%)$

$83(55.1 \%)$

$31.68 \pm 7.37$

$86.71 \pm 16.72$

$116.37 \pm 15.14$

$78.83 \pm 11.26$

$15(10-24)$

$96.35 \pm 29.4$

$4.71 \pm 4.11$

$5.3(3.7-8.7)$

$7.4(4.9-14.4)$

77 (33.4-155)

13.5 (1-57.6)

$2.6 \pm 2.0$

$21.5 \pm 14.17$

\section{TABLE 1: Baseline characteristics and biochemical parameters of study participants}

HOMA-IR, homeostatic model assessment of insulin resistance; FSH, follicle stimulating hormone; LH, luteinizing hormone; TSH, thyroid stimulating hormone; IQR, interquartile range.

Data is presented as mean $\pm \mathrm{SD}$ or $\mathrm{n}(\%)$ or median (IQR).

The most common clinical presentation of women with PCOS was menstrual irregularity; oligomenorrhea was reported in 61 (39.85\%) and amenorrhea in 44 (38.9\%), followed by hirsutism that was noted in 80 (52.3\%) while acne and alopecia were found in 33 (21.5\%) and 24 (15.6\%), respectively. Moreover, infertility was present in 50 (32.6\%) women, whereas 51 (33.3\%) women had polycystic morphology of ovaries on ultrasound. The family history of diabetes, hypertension, dyslipidemia and obesity was present in 85 (55.6\%), 65 (42.5\%), 22 (14.4\%), and 44 (28.8\%) participants, respectively, as mentioned in Table 2. 


\section{Cureus}

\begin{tabular}{|c|c|c|}
\hline Parameters & Frequency (n) & Percentage \\
\hline $\mathrm{N}$ & 153 & - \\
\hline Menstrual cycle irregularity & 89 & 58.2 \\
\hline Dysmenorrhea & 41 & 26.7 \\
\hline Oligomenorrhea & 61 & 39.8 \\
\hline Amenorrhea & 44 & 28.7 \\
\hline Dysfunctional uterine bleeding & 17 & 11.2 \\
\hline Infertility & 50 & 32.6 \\
\hline Hirsutism & 80 & 52.3 \\
\hline Alopecia & 24 & 15.6 \\
\hline Acne & 33 & 21.5 \\
\hline Polycystic ovaries on ultrasound (PCOM) & 51 & 33.3 \\
\hline
\end{tabular}

\section{TABLE 2: Clinical presentations of study participants}

PCOM, polycystic ovarian morphology.

Data is presented as $n(\%)$.

The frequency and percentages of MetS and its individual parameters are shown in Figure 1. Out of 153 women with PCOS, 71 (46.4\%) participants fulfilled the criteria of MetS. The obesity was noted in the highest frequency, in 126 (82.4\%), followed by dyslipidemia, in 86 (56.2\%), whereas 48 (31\%) females had hypertension and 40 (26\%) were either diagnosed with diabetes or impaired glucose tolerance.

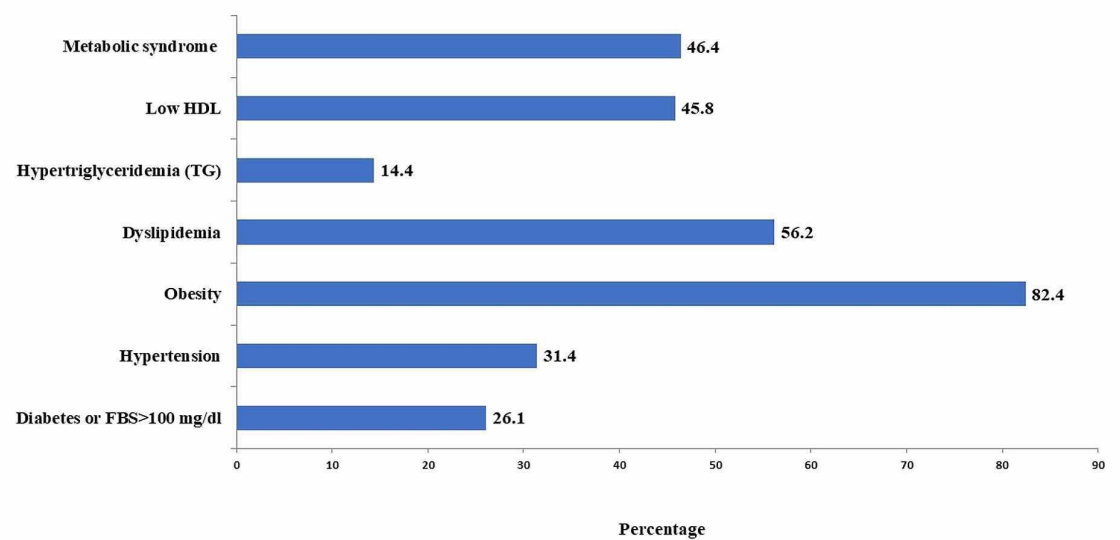

FIGURE 1: Frequency of metabolic syndrome in study participants HDL, high-density lipoprotein; FBS, fasting blood sugar.

Age, BMI, waist circumference and blood pressure had statistically significant association in PCOS women with MetS. The comparison among PCOS women with and without MetS in terms of baseline characteristics and lab parameters is shown in Table 3. 


\section{Cureus}

\begin{tabular}{|c|c|c|c|}
\hline Variables & Without MetS & With MetS & P-value \\
\hline $\mathrm{N}$ & 82 & 71 & - \\
\hline Age (years) & $25.63 \pm 7.5$ & $29.01 \pm 8.5$ & 0.01 \\
\hline Age at menarche (years) & $12.82 \pm 1.21$ & $12.51 \pm 1.17$ & 0.146 \\
\hline \multicolumn{4}{|l|}{ Marital status } \\
\hline Single & $37(44.21 \%)$ & $28(39.4 \%)$ & \multirow{2}{*}{0.291} \\
\hline Married & $40(48.7 \%)$ & $43(60.6 \%)$ & \\
\hline Not known & $5(6.1 \%)$ & $0(0 \%)$ & \\
\hline BMI $\left(\mathrm{kg} / \mathrm{m}^{2}\right)$ & $28.64 \pm 7.12$ & $34.89 \pm 6.2$ & $<0.0001$ \\
\hline Walst circumerence (cm) & $75.9 / \pm 1 / .99$ & $93.29 \pm 11.9$ & $<0.0001$ \\
\hline Systolic blood pressure (mmHg) & $110.3 \pm 12.55$ & $122.43 \pm 15.16$ & $<0.0001$ \\
\hline Diastolic blood pressure (mmHg) & $74.16 \pm 8.62$ & $83.9 \pm 11.64$ & $<0.0001$ \\
\hline HOMA & $4.47 \pm 3.58$ & $4.88 \pm 4.48$ & 0.693 \\
\hline Fasting blood glucose (mg/dl) & $89.73 \pm 10.57$ & $101.06 \pm 36.85$ & 0.073 \\
\hline Fasting insulin & $14(9.2-19.8)$ & $16.5(10.6-25.25)$ & 0.505 \\
\hline FSH & $5.4(4.2-8.7)$ & $5.1(3.5-8.5)$ & 0.592 \\
\hline LH & $7.2(5.1-21.0)$ & $7.5(4.8-12.6)$ & 0.367 \\
\hline Estradiol & $74(31.2-80)$ & $165(35-242)$ & 0.222 \\
\hline Testosterone & $20(1-62.25)$ & $7.5(1.75-39.75)$ & 0.782 \\
\hline TSH & $2.4 \pm 1.76$ & $2.7 \pm 2.28$ & 0.616 \\
\hline Prolactin & $24.43 \pm 17.15$ & $19.65 \pm 11.86$ & 0.254 \\
\hline
\end{tabular}

\section{TABLE 3: Comparison of different variables in PCOS women with and without MetS}

PCOS, polycystic ovary syndrome; MetS, metabolic syndrome; HOMA, homeostatic model assessment; FSH, follicle stimulating hormone; LH, luteinizing hormone; TSH, thyroid stimulating hormone; IQR, interquartile range.

Data is presented as mean \pm SD or $n(\%)$ or median (IQR). P-value $<0.05$ was considered to be statistically significant.

As far as the individual component of MetS is concerned, diabetes was more prevalent in family members of women with PCOS followed by hypertension, obesity and dyslipidemia as shown in Figure 2. 


\section{Cureus}

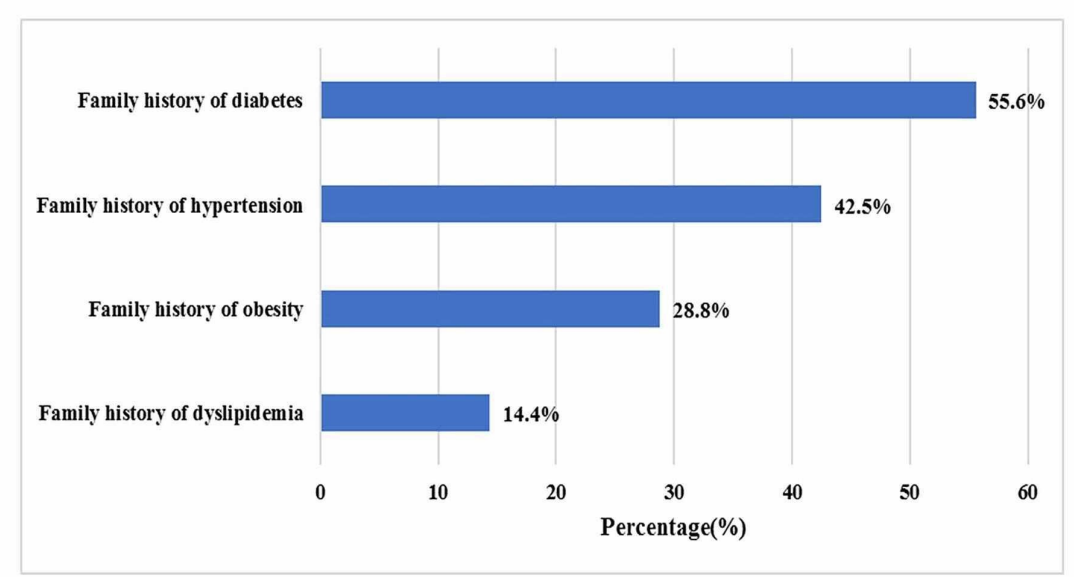

FIGURE 2: The components of metabolic syndrome in family members of women with PCOS

PCOS, polycystic ovary syndrome.

\section{Discussion}

The present study demonstrated a high frequency of MetS in women with PCOS. Among the different parameters of MetS, obesity and dyslipidemia were the two most frequent components in our study participants, while diabetes and hypertension were reported more in family members of the recruited women. The most common presenting complaint was menstrual irregularity (oligomenorrhea, amenorrhea) followed by hirsutism in our study participants.

The manifestation of MetS and clustering of its component varies in relation to studied population, ethnicity, and criteria applied to identify the MetS $[12,15]$. We observed MetS in almost half of our study participants; a similar finding was observed by Ehrmann et al., who reported a prevalence of 33.4\% [15]. However, in contrast to our finding, MetS was noted only in $11.9 \%$ participants in another study [16].

Metabolic derangements are more obvious in obese as compared to non-obese PCOS counterparts [9]. Twothirds of our study participants were obese; a similar prevalence was reflected in other studies [1,17]. In agreement with our observation, Anjum et al. [12] and Ehrmann et al. [15] observed that MetS and its distinct components are particularly common among females with increased BMI. However, Akram and Roohi [18] and Shanmugham et al. [19] reported obesity in only one-third of their study participants; this may be explained by the difference in the prevalence of obesity in different parts of the world [1]. The reported prevalence of obesity in Pakistan was $62.1 \%$ as per the second National Diabetes Survey of Pakistan (NDSP) during year 2016-2017 [20]. This high prevalence of obesity may be responsible for the increased frequency of MetS in our study population.

Women with PCOS, especially obese females, frequently displayed atherogenic dyslipidemia [10]. More than half of our study participants had dyslipidemia; the same was noted in another study [21]. In our study, low HDL contributed more as compared to high triglycerides to dyslipidemia, whereas Celik et al. [22] revealed significant high triglyceride levels in PCOS women as compared to controls. This disparity in data is enlightened by variation in the life style, dietary habits, prevalence of diabetes and obesity in different communities [10].

One third of our study participants were hypertensive, and similar finding was observed in another study [23]. In contrast to our finding, another study reported the frequency of hypertension in $10.7 \%$ of study participants [18]. Women with PCOS are at an increased risk of developing dysglycemia due to IR [5]. One fourth of our study participants had either impaired fasting plasma glucose or diabetes. Similar findings were revealed by other studies as well [24,25]. However, in contrast to our findings, Amato et al. noted impaired fasting glucose/T2DM in $12 \%$ of women with PCOS [26]; the observed diversity may be due to the variance in the prevalence of diabetes in different parts of the world.

The increased occurrence of different components of MetS among family members of women with PCOS pointed towards the genetic and environmental factors behind its pathogenesis. The significantly increased prevalence of components of MetS in the family history of our study participants is comparable with other studies also $[18,27,28]$. 
Menstrual cycle irregularities are noted early because of their monthly occurrence and may be the reason for being the common presenting complaint to health care professionals. In this study, menstrual irregularity was the main presenting complaint in the majority of study participants. This was also reflected in other studies $[1,19,26]$. In contrast to this finding, a meta-analysis revealed infertility and hirsutism as common presenting problem in females with PCOS [2].

Our study demonstrated hirsutism in almost half of PCOS women, and the same frequency was perceived by Amato et al. [26]. However, in contrast to our observation, Najem et al. [17] and Shanmugam et al. [19] found hirsutism in above $90 \%$ of study participants, whereas several other studies concluded that hirsutism affects $65 \%-75 \%$ of Southeast Asian women [1,21]. Statistics advocated that androgen level and hair follicle sensitivity to androgen are responsible for variations in the severity of the hirsutism seen in women with PCOS belonging to different ethnicities [5]. The number of women seeking medical advice regarding hirsutism varies widely depending upon the socio-cultural norms and acceptability in society.

Furthermore, a lot of women use herbal products and home remedies for hirsutism that may result in the variation of reported frequency of hirsutism.

Studies postulated that IR and compensatory hyperinsulinemia are common pathogenic factors for both PCOS and MetS [26]. In our study, IR was noted in two-thirds of women with PCOS and this finding is consistent with the research performed by Yu and Wangl [21]. Several other case control studies reported statistically significant high fasting insulin levels and HOMA-IR in women with PCOS [11,22,24]. However, this observation was contradictory to a study that showed IR in only one third participants [29]. The variation in genetic and environmental factors may be the reason behind the difference in severity of IR in different studied population [26]. Polycystic ovarian morphology (PCOM) on ultrasound was observed in one third of females with PCOS. In contrast to our findings, other studies demonstrated PCOM in more than 90 $\%$ of participants [19]. It is a well-known fact that ovarian androgenic dysfunction ranges from subclinical hyperandrogenemia in normal-variant PCOM to severe ovarian hyperandrogenism in most classic PCOS [3]. Moreover, polycystic ovaries are common in anovulatory women due to several causes and are not necessarily associated with PCOS [30].

\section{Strength and limitation}

This study provides local data on MetS in women with PCOS that would be helpful for further studies; however, this being a single-center study is the main limitation. Further studies are required to validate our findings.

\section{Conclusions}

The frequency of MetS is high in females presenting with PCOS, and therefore, metabolic surveillance should be considered in such women to reduce the risk of potential complications.

\section{Additional Information \\ Disclosures}

Human subjects: Consent was obtained by all participants in this study. Institutional Review Board, Baqai Institute of Diabetology and Endocrinology issued approval Ref:/BIDE/IRB/SANJUM/08/19/022. Animal subjects: All authors have confirmed that this study did not involve animal subjects or tissue. Conflicts of interest: In compliance with the ICMJE uniform disclosure form, all authors declare the following: Payment/services info: All authors have declared that no financial support was received from any organization for the submitted work. Financial relationships: All authors have declared that they have no financial relationships at present or within the previous three years with any organizations that might have an interest in the submitted work. Other relationships: All authors have declared that there are no other relationships or activities that could appear to have influenced the submitted work.

\section{References}

1. Sinha U, Sinharay K, Saha S, Longkumer TA, Baul SN, Pal SK: Thyroid disorders in polycystic ovarian syndrome subjects: a tertiary hospital based cross-sectional study from Eastern India. Indian J Endocrinol Metab. 2013, 17:304-309. 10.4103/2230-8210.109714

2. Goodman NF, Cobin RH, Futterweit W, Glueck JS, Legro RS, Carmina E: American Association of Clinical Endocrinologists, American College of Endocrinology, and Androgen Excess and PCOS Society disease state clinical review: guide to the best practices in the evaluation and treatment of polycystic ovary syndrome part 1. Endocr Pract. 2015, 21:1291-300. 10.4158/EP15748.DSC

3. Rosenfield RL: The polycystic ovary morphology - polycystic ovary syndrome spectrum . J Pediatr Adolesc Gynecol. 2015, 28:412-419. 10.1016/j.jpag.2014.07.016

4. De Sousa SM, Norman RJ: Metabolic syndrome, diet and exercise. Best Pract Res Clin Obstet Gynaecol. 2016, 37:140-151. 10.1016/j.bpobgyn.2016.01.006

5. Ramanand SJ, Ghongane BB, Ramanand JB, Patwardhan MH, Ghanghas RR, Jain SS: Clinical characteristics of polycystic ovary syndrome in Indian women. Indian J Endocrinol Metab. 2013, 17:138-145. 10.4103/22308210.107858 
6. Wang R, Mol BW: The Rotterdam criteria for polycystic ovary syndrome: evidence-based criteria? . Hum Reprod. 2017, 32:261-264. 10.1093/humrep/dew287

7. Imam SK, Shahid SK, Hassan A, Alvi Z: Frequency of the metabolic syndrome in type 2 diabetic subjects attending the diabetes clinic of a tertiary care hospital. J Pak Med Assoc. 2007, 57:239-242.

8. Poppe K, Velkeniers B, Glinoer D: Thyroid disease and female reproduction. Clin Endocrinol (Oxf). 2007, 66:309-321. 10.1111/j.1365-2265.2007.02752.x

9. Cheung LP, Ma RC, Lam PM, et al.: Cardiovascular risks and metabolic syndrome in Hong Kong Chinese women with polycystic ovary syndrome. Hum Reprod. 2008, 23:1431-1438. 10.1093/humrep/den090

10. Romanowski MD, Parolin MB, Freitas AC, Piazza MJ, Basso J, Urbanetz AA: Prevalence of non-alcoholic fatty liver disease in women with polycystic ovary syndrome and its correlation with metabolic syndrome. Arq Gastroenterol. 2015, 52:117-123. 10.1590/S0004-28032015000200008

11. Ali AT: Polycystic ovary syndrome and metabolic syndrome. Ceska Gynekol. 2015, 80:279-289.

12. Anjum N, Zohra S, Arif A, Azhar A, Qureshi M: Prevalence of metabolic syndrome in Pakistani women with polycystic ovarian syndrome. Pak J Biochem Mol Biol. 2013, 46:97-100.

13. Bañuls C, Rovira-Llopis S, de Marañon AM, et al.: Metabolic syndrome enhances endoplasmic reticulum, oxidative stress and leukocyte-endothelium interactions in PCOS. Metabolism. 2017, 71:153-162. 10.1016/j.metabol.2017.02.012

14. Herrera-Enriquez K, Narvaez-Guerra O: Discordance of metabolic syndrome and abdominal obesity prevalence according to different criteria in Andean highlanders: a community-based study. Diabetes Metab Syndr. 2017, 11:S359-S364. 10.1016/j.dsx.2017.03.016

15. Ehrmann DA, Liljenquist DR, Kasza K, Azziz R, Legro RS, Ghazzi MN, PCOS/Troglitazone Study Group: Prevalence and predictors of the metabolic syndrome in women with polycystic ovary syndrome . J Clin Endocrinol Metab. 2006, 91:48-53. 10.1210/jc.2005-1329

16. Lerchbaum E, Schwetz V, Giuliani A, Obermayer-Pietsch B: Hypertriglyceridemic waist is associated with impaired glucose tolerance in polycystic ovary syndrome. Nutr Metab Cardiovasc Dis. 2013, 23:E15-E16. 10.1016/j.numecd.2012.11.004

17. Najem F, Elmehdawi R, Swalem A: Clinical and biochemical characteristics of polycystic ovary syndrome in Benghazi-Libya: a retrospective study. Libyan J Med. 2008, 3:71-74. 10.4176/080122

18. Akram M, Roohi N: Endocrine correlates of polycystic ovary syndrome in Pakistani women . J Coll Physicians Surg Pak. 2015, 25:22-26.

19. Shanmugham D, Natarajan S, Karthik A: Prevalence of thyroid dysfunction in patients with polycystic ovarian syndrome: a cross sectional study. Int J Reprod Contracept Obstet Gynecol. 2018, 7:3055-3059. 10.18203/2320-1770.ijrcog20182951

20. Basit A, Fawwad A, Qureshi H, Shera AS, NDSP Members: Prevalence of diabetes, pre-diabetes and associated risk factors: second National Diabetes Survey of Pakistan (NDSP), 2016-2017. BMJ Open. 2018, 8:e020961. 10.1136/bmjopen-2017-020961

21. Yu Q, Wang JB: Subclinical hypothyroidism in PCOS: impact on presentation, insulin resistance, and cardiovascular risk. Biomed Res Int. 2016, 2016:2067087. 10.1155/2016/2067087

22. Celik C, Abali R, Tasdemir N, Guzel S, Yuksel A, Aksu E, Yılmaz M: Is subclinical hypothyroidism contributing dyslipidemia and insulin resistance in women with polycystic ovary syndrome?. Gynecol Endocrinol. 2012, 28:615-618. 10.3109/09513590.2011.650765

23. Quiñónez CZ, Silva RR, Torres JJ: Obesity, arterial hypertension, metabolic disorders, and polycystic ovary syndrome. (Article in Spanish). Ginecol Obstet Mex. 2000, 68:317-322.

24. Legro RS, Kunselman AR, Dodson WC, Dunaif A: Prevalence and predictors of risk for type 2 diabetes mellitus and impaired glucose tolerance in polycystic ovary syndrome: a prospective, controlled study in 254 affected women. J Clin Endocrinol Metab. 1999, 84:165-169. 10.1210/jcem.84.1.5393

25. Shi Y, Guo M, Yan J, et al.: Analysis of clinical characteristics in large-scale Chinese women with polycystic ovary syndrome. Neuro Endocrinol Lett. 2007, 28:807-810.

26. Amato MC, Galluzzo A, Merlino S, Mattina A, Richiusa P, Criscimanna A, Giordano C: Lower insulin sensitivity differentiates hirsute from non-hirsute Sicilian women with polycystic ovary syndrome. Eur J Endocrinol. 2006, 155:859-865. 10.1530/eje.1.02290

27. Zahiri Z, Sharami SH, Milani F, Mohammadi F, Kazemnejad E, Ebrahimi H, Heirati SF: Metabolic syndrome in patients with polycystic ovary syndrome in Iran. Int J Fertil Steril. 2016, 9:490-496. 10.22074/ijfs.2015.4607

28. Yilmaz B, Vellanki P, Ata B, Yildiz BO: Metabolic syndrome, hypertension, and hyperlipidemia in mothers, fathers, sisters, and brothers of women with polycystic ovary syndrome: a systematic review and metaanalysis. Fertil Steril. 2018, 109:356-364.E32. 10.1016/j.fertnstert.2017.10.018

29. Tabassum R, Imtiaz F, Sharafat S: Prevalence and clinical profile of insulin resistance in young women of poly cystic ovary syndrome: a study from Pakistan. Pak J Med Sci. 2013, 29:593-596. 10.12669/pjms.292.3180

30. Adams J, Polson DW, Franks S: Prevalence of polycystic ovaries in women with anovulation and idiopathic hirsutism. Br Med J (Clin Res Ed). 1986, 293:355-359. 10.1136/bmj.293.6543.355 